\title{
COVID-19 testing turns to T cells
}

\author{
A diagnostic test based on sequencing long-lived SARS-CoV-2-specific \\ memory $T$ cells provides a complement to antibody testing for determining \\ previous exposure to SARS-CoV-2.
}

F ollowing last month's US Food and Drug Administration (FDA) Emergency Use Authorization for Adaptive Biotechnologies' T-Detect COVID-19 test, routine T-cell testing has entered a new era. The Adaptive test involves laboratory-based next-generation sequencing to identify $\mathrm{T}$ cells that recognize SARS-CoV-2 antigens. The test is not intended for the diagnosis of active infection but is a complement to antibody tests used to confirm recent or previous infections. The lab-based procedure, which has a seven- to ten-day turnaround time, is now authorized for use on samples taken from individuals at least 15 days after the onset of symptoms.

Increasing interest is focused on the role of T-cell immunity in fighting SARS-CoV-2 infection and in providing resistance to re-infection. A new analysis of T cells from people who recovered from COVID-19 has confirmed that they remain active against three of the new SARS-CoV-2 variants of concern: B1.1.7, B.351 and B.1.1.248. The study, conducted by a team from the US National Institute of Allergy and Infectious Diseases (NIAID), Johns Hopkins University School of Medicine, Johns Hopkins Bloomberg School of Public Health and Singapore-based biotech company ImmunoScape, will further boost confidence that the efficacy of vaccines developed against the original pandemic strain will not be overly compromised as these new variants-and others-spread more widely.

Until now, researchers have mostly relied on the use of lateral flow assay or enzyme-linked immunosorbent assay (ELISA) tests for SARS-CoV-2 antibodies to determine whether a person has been exposed to the virus. Understanding the neutralizing antibody response has been considered central to establishing protection against the virus. "It's easy to test," says Andrew Redd of NIAID, who led the recent study. Although critical, antibodies are part of a larger and incompletely understood set of humoral and cellular immune responses, which has received little attention. These include additional antibody functions, such

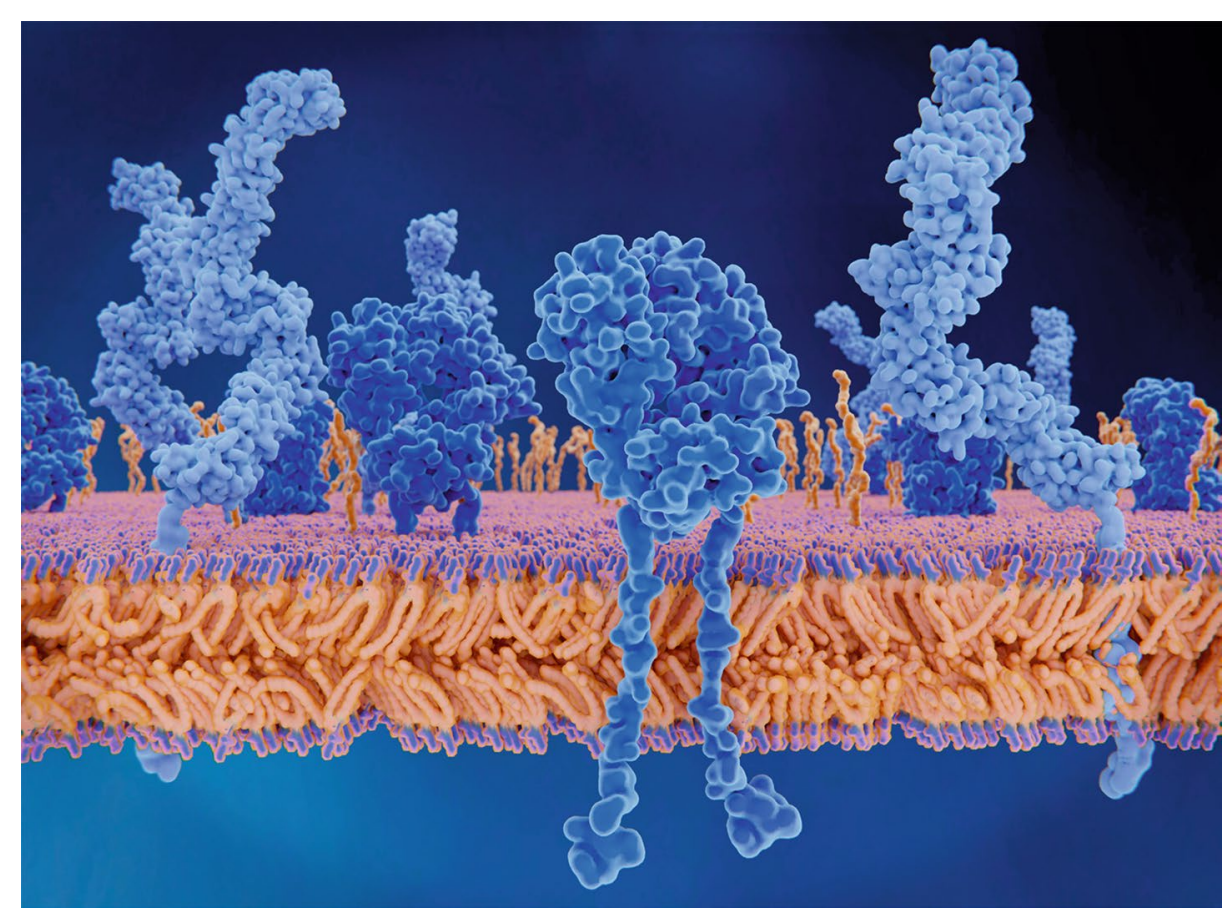

\section{What two US counties will tell us about COVID-19}

A rapid-testing program will be rolled out across two communities to ask whether widespread at-home tests are effective at curbing community spread. The 'Say Yes! COVID Test' initiative will enroll up to 160,000 volunteers in Tennessee and North Carolina, who will self-administer three rapid, at-home tests a week. The testing program, launched by the Centers for Disease Control and Prevention (CDC) and the National Institutes of Health (NIH), aims to understand whether frequent tests can reduce viral transmission during this and future pandemics. NIH Director Francis S. Collins says "this is the first initiative of this scale to make free, rapid, self-administered tests available community-wide" to determine such tests' effectiveness. The test is the QuickVue test from Quidel, an at-home diagnostic that detects SARS-CoV-2 antigen and provides a result in ten minutes from a nasal swab. The test received Emergency Use Authorization on 1 March 2021 and is supplied through Rapid Acceleration of Diagnostics (RADx), an NIH program set up to fund innovative COVID-19 testing technologies. Antigen tests such as QuickVue are less sensitive than PCR, which is the gold standard for diagnosing COVID-19. If used frequently, however, such at-home tests could rapidly identify people who are asymptomatic but still infectious, encouraging them to self-isolate. Such scaled-up testing could potentially stop viral spread, but data to show this is the case has not been forthcoming. "All the mathematical models predict that. But this is a real-world, real-life example," said Bruce Tromberg, director of the National Institute of Biomedical Imaging and Bioengineering, in The New York Times. Tromberg is leader of the RADx Tech program.

Published online: 12 May 2021 https://doi.org/10.1038/s41587-021-00931-6 


\section{Barista milk: climate- friendly and still dairy}

A climate-friendly barista milk from cows that emit less methane has launched in selected UK coffee shops. The milk comes from livestock fed Mootral Ruminant, a cattle feed supplement by the Swiss-British agritech company Mootral. The food additive, developed by a team of veterinary researchers from Belgium, Denmark, Finland, Germany, Ireland, Japan, the Netherlands, the United Kingdom and the United States, cuts enteric methane emissions by up to $38 \%$. It builds on the fact that microbial communities in ruminants' digestive systems ferment crude plant fiber to short-chain fatty acids, which the cow takes up through its gut. This fermentation, however, also produces methane, a major contributor of greenhouse gases. When added to feed, Mootral changes cows' gut methanogen content and microbial metabolic pathways to reduce methane in their burps. The supplement is a mix of garlic-known for the organosulfur compound allicin, which has antimicrobial propertiesand flavonoids extracted from bitter oranges. In lab experiments, a team at the University of Veterinary Medicine in Hanover, Germany, found Mootral altered the composition of the microbial community of Archaea, the main producers of methane during rumen fermentation. In a farm study conducted in the Netherlands, Mootral boosted the cows' milk yield by $4 \%$ and increased fat content too, with no changes to the milk's taste and appearance. In Scotland, a commercial dairy farm with 400 dairy cows found that Mootral reduced methane emissions by $30 \%$ on average as measured with a hand-held methane laser detector. Another trial, conducted at the University of California Davis, measured a $23 \%$ drop in methane production. Thomas Hafner, CEO and cofounder of Mootral, says in a press release: "It's time for us to help cows become part of the solution in the global fight against climate change." Mootral next plans to focus on climate-friendly beef.

Published online: 12 May 2021

https://doi.org/10.1038/s41587-021-00930-7 as antibody-dependent cellular cytotoxicity, complement activation and phagocyte recruitment. Unravelling their contribution to SARS-CoV-2 immunity is an ongoing challenge. "There are assays to do that, it's just complicated to do," says Redd.

The same can be said for assaying T-cell-mediated immunity. The NIAID study relied on a complex laboratory test to identify T-cell epitopes specific to SARS-CoV-2, employing a combination of mass cytometry and combinatorial staining of peptide-major histocompatibility complex (MHC)-bound tetramers. The complexity of the assay and data generated necessarily confine the assay to use in specialist laboratories. "The data that it generates are massive. The analysis side of it is a big lift," Redd says.

The Adaptive Biotechnologies sequencing test provides a simpler option. In a clinical validation study, the T-Detect COVID assay attained $97.1 \%$ sensitivity (defined as positive percent agreement) and $100 \%$ specificity (defined as negative percent agreement) as compared with PCR testing from 15 days or more after diagnosis.

The test emerged from a longstanding collaboration between Adaptive and Microsoft to apply machine learning to define the 'rules' according to which T-cell receptors (TCRs) identify their cognate antigens. That, combined with extensive genomic characterization of the T-cell repertoires of people infected with SARS-CoV-2, allowed Adaptive to define a broad set of TCRs that are indicative of infection with the virus. The test sequences the total TCR repertoire present in a given sample and then calculates the relative enrichment for SARS-CoV-2-specific TCRs compared with predefined thresholds to determine the result, taking into account variation in individuals' immune responses.

Adaptive's test analyzes both the frequency and distribution of the TCRs that are present and the T-cell clonal expansions that have occurred. "The algorithm takes into account clonal breadth and depth," says Lance Baldo, chief medical officer at Adaptive.

Traditional T-cell testing methods, such as enzyme-linked immunosorbent spot (ELISpot) and intracellular cytokine staining, require scientists to directly measure T cells' cytokine production following antigen stimulation under specific conditions. "Live cells don't do well out of the body for very long times," says Baldo. "We're looking at the genomic DNA of T cells, which is a very stable analyte." Samples can be shipped at room temperature and frozen for long periods. The new test is also more accurate than traditional methods that only allow a small number of peptide antigens to be measured simultaneously. And because T-cell responses last longer than antibody responses, the test also provides a wider window for establishing whether or not an individual has been infected.

One immediate application of the test will be to monitor the effectiveness of the various vaccines that are being rolled out, particularly in vulnerable populations. "We don't know yet how long the immunity is going to last," says Joaquín Martínez-Lopez of Spain's National Cancer Research Center in Madrid, who collaborated with Adaptive on the development of the test.

Qiagen, of Hilden, Germany, is also developing a next-generation sequencingbased T-cell diagnostic, having signed an agreement with TScan Therapeutics, which will provide it with access to the latter's intellectual property and associated data for several panels of epitopes in SARS-CoV-2 that are recognized by $\mathrm{CD} 8^{+} \mathrm{T}$ cells from patients recovering from COVID-19. TScan scientists have identified three to eight epitopes for each of the six most common human leukocyte antigen types in people convalescing from COVID-19.

\section{Cormac Sheridan \\ Dublin, Ireland}

Published online: 12 May 2021

https://doi.org/10.1038/s41587-021-00920-9 